\title{
Characteristics of Electrospray-Ionization Detection of Synthetic Di- to Penta- Oligopeptides by Amine Derivatizations
}

\author{
Weilin Shen, Kiyomi Ono, Mitsuru TanaKa, and Toshiro Matsui ${ }^{\dagger}$ \\ Department of Bioscience and Biotechnology, Faculty of Agriculture, Graduate School of Kyushu University, \\ 744 Motooka, Fukuoka 819-0395, Japan
}

\begin{abstract}
Chemical derivatizations have been extensively developed for highly sensitive detection of bioactive small peptides; however, their advantages from the viewpoint of longer oligopeptides remain unverified. In this study, electrosprayionization (ESI)-mass spectrometric (MS) detection of synthetic di- to pentapeptides consisting of glycine and sarcosine were characterized by four amine derivatization methods. It was concluded that the ESI-MS detection of di- to pentapeptides was characterized by the molecular surface area of derivatized peptide moieties with an optimal value of $250-300 \AA^{2}$, regardless of hydrophobicity and derivatization methods.
\end{abstract}

Keywords Oligopeptide, ESI-MS, chemical derivatization, molecular surface area

(Received March 30, 2021; Accepted May 10, 2021; Advance Publication Released Online by J-STAGE May 14, 2021)

\section{Introduction}

Food derived small peptides have been demonstrated to possess various bioactivities and are believed to be beneficial for the prevention of metabolic syndrome. ${ }^{1}$ Upon oral intake, the functionality of such bioactive peptides depends on their ability to penetrate the intestinal barrier and reach the target organs. ${ }^{2}$ Thus, bioavailability evaluation is becoming critical in understanding biofunctions, and development of highly sensitive and reliable analytical methods is essential. ${ }^{3}$

For highly sensitive and quantitative analysis of smaller diand tripeptides, several chemical derivatization methods have been developed for high-performance liquid chromatography (HPLC). Fluorescence derivatization of a dipeptide Val-Tyr with naphthalene-2,3-dialdehyde (NDA) enabled detection at the fmol level. ${ }^{4-6}$ The emergence of mass spectrometry (MS) enabled detection with higher selectivity. Nonetheless, a lower detection limit (LOD) is desirable. With an electrospray ionization (ESI) ion source, the aim of derivatization is to increase the hydrophobicity of the targeted peptides to increase the ionization efficiency. Therefore, several derivatization methods have been developed to enhance the ESI-MS signal of small polar amines over the decades $;{ }^{7}$ among them, we reported the use of 2,4,6-trinitrobenzene sulfonate (TNBS) as an efficient derivatization reagent for primary amines. ${ }^{8}$ TNBS derivatization resulted in improved detection of diverse dipeptides by a factor of 3-55 fold $(S / N)$ and proved applicable in peptide bioavailability studies using biofluids such as rat plasma., ${ }^{9,10}$ A recent report identified longer oligopeptides (3-8 amino acid residues) in rat plasma after the ingestion of $\beta$-conglycinin protein,${ }^{11}$ suggesting that longer peptides could also be absorbed and play a role in biological regulation. While chemical

$\dagger$ To whom correspondence should be addressed.

E-mail: tmatsui@agr.kyushu-u.ac.jp derivatizations have been applied to increase the detection of di- and tripeptides, their benefits on longer oligopeptides remain unverified. Here, we investigated the ESI-MS detection of dito pentapeptides in combination with four reported amine derivatization reagents, namely, NDA, TNBS, $N$-succinimidyl 7-methoxycoumarin-3-carboxylate (7MeO-Cou), and 3-aminopyridyl- $N$-hydroxysuccinimidyl carbamate (APDS) (Fig. 1). The peptides used herein were synthetic oligopeptides consisting of glycine (Gly, G) and sarcosine (Sar, S) to exclude the influence of amino acid residues on MS detection.

\section{Experimental}

\section{Chemicals and reagents}

GG, GGG, and TNBS were purchased from Nacalai Tesque (Kyoto, Japan). GS, 7MeO-Cou, and NDA were purchased from Sigma-Aldrich (St. Louis, MO, USA). GSS was obtained from Bachem (Bubendorf, Switzerland). GSSS and GSSSS were obtained from Biomatik (Cambridge, ON, Canada). APDSTAG $^{\circledR}$ was purchased from Wako (Osaka, Japan). GGGG and GGGGG were synthesized inhouse using Fmoc-Gly-OH and Fmoc-Gly-Wang resin (100-200 mesh), respectively, obtained from Kokusan Chemicals (Tokyo, Japan). All the other chemicals were of analytical reagent grade and were used without further purification.

\section{Chemical derivatization of peptides}

Herein, four types of chemical derivatizations were conducted for the targeted peptides, according to previous reports with

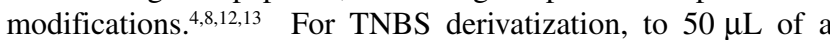
standard peptide solution, $50 \mu \mathrm{L}$ of $150 \mathrm{mmol} / \mathrm{L}$ TNBS in $100 \mathrm{mmol} / \mathrm{L}$ borate buffer ( $\mathrm{pH} \mathrm{8.0)}$ was added. After incubation at $30^{\circ} \mathrm{C}$ for $30 \mathrm{~min}, 100 \mu \mathrm{L}$ of $0.2 \%$ formic acid (FA) was added to stop the reaction. For APDS derivatization, to $195 \mu \mathrm{L}$ of a peptide solution dissolved in $100 \mathrm{mmol} / \mathrm{L}$ borate buffer $(\mathrm{pH} 8.0$ ), 
(A) TNBS derivatization<smiles>CCNc1c([N+](=O)[O-])cc([N+](=O)[O-])cc1[N+](=O)[O-]</smiles>

(B) APDS derivatization<smiles>CCCCCCCCCCCCC(=O)Nc1cccnc1</smiles>

(C) 7MeO-Cou derivatization<smiles>CCNC(=O)c1cc2ccc(OC)cc2oc1=O</smiles>

(D) NDA derivatization<smiles>Cn1cc2cc3ccccc3cc2c1C#N</smiles>

Fig. 1 Structures of peptide derivatives of (A) TNBS, (B) APDS, (C) 7MeO-Cou, and (D) NDA.

$5 \mu \mathrm{L}$ of $20 \mathrm{mg} / \mathrm{mL}$ APDS in acetonitrile was added. The mixture was incubated at $60^{\circ} \mathrm{C}$ for $30 \mathrm{~min}$. For $7 \mathrm{MeO}-\mathrm{Cou}$ derivatization, to $20 \mu \mathrm{L}$ of a standard peptide solution, $10 \mu \mathrm{L}$ of $1 \mathrm{~mol} / \mathrm{L}$ tetraethylammonium bromide and $10 \mu \mathrm{L}$ of $30 \mu \mathrm{mol} / \mathrm{L}$ $7 \mathrm{MeO}-\mathrm{Cou}$ were added. The mixture was incubated at $30^{\circ} \mathrm{C}$ for $30 \mathrm{~min}$ for reaction. For NDA derivatization, to $50 \mu \mathrm{L}$ of a peptide solution dissolved in $100 \mathrm{mmol} / \mathrm{L}$ borate buffer $(\mathrm{pH} 8.0)$, $10 \mu \mathrm{L}$ of $1 \mathrm{mmol} / \mathrm{L}$ sodium cyanide and $40 \mu \mathrm{L}$ of $0.1 \mathrm{mmol} / \mathrm{L}$ NDA dissolved in $\mathrm{MeOH}$ were added. The mixture was incubated at $30^{\circ} \mathrm{C}$ for $50 \mathrm{~min}$ for reaction. The reactions were performed to obtain peptide derivatives at a final concentration of $0.5 \mu \mathrm{mol} / \mathrm{L}$.

\section{LC-ESI-MS analysis}

Chromatographic separation was performed using an Agilent 1200 series (Agilent, Waldbronn, Germany) on a Cosmosil $5 \mathrm{C}_{18}$-MS-II column $(2.0 \times 150 \mathrm{~mm}$, Nacalai Tesque $)$ at $40^{\circ} \mathrm{C}$ with a linear gradient elution of methanol $(\mathrm{MeOH})$ containing $0.1 \%$ formic acid $(0-100 \%$ over $20 \mathrm{~min})$ at a flow rate of $0.2 \mathrm{~mL} / \mathrm{min}$. An injection volume of $20 \mu \mathrm{L}$ was used herein. ESI-MS analysis was performed using a micrOTOF II (Bruker Daltonics, Bremen, Germany) in positive ion mode. The MS conditions were as follows: drying gas, $\mathrm{N}_{2}$; flow rate, $8.0 \mathrm{~L} / \mathrm{min}$; drying gas temperature, $200^{\circ} \mathrm{C}$; drying gas pressure, $1.6 \mathrm{bar}$;

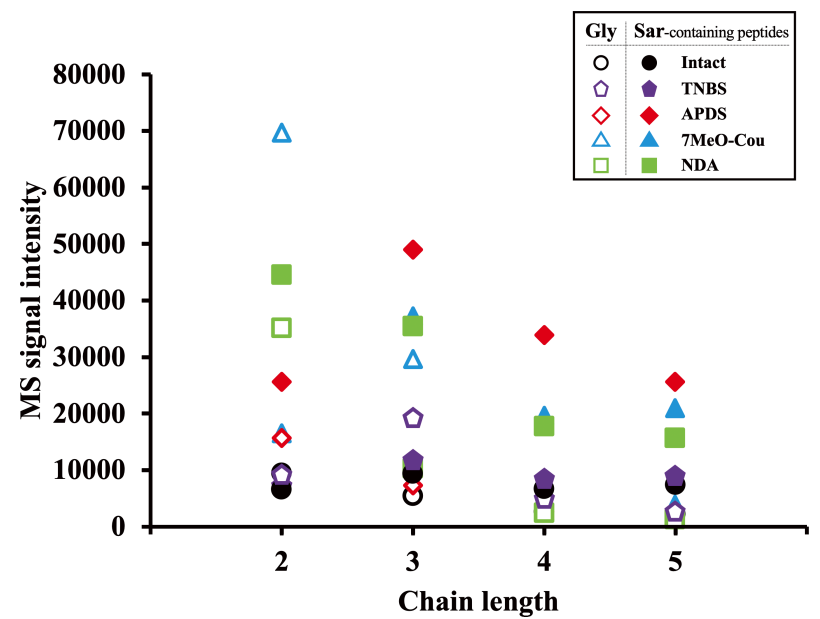

Fig. 2 MS signal intensities of 40 peptides and derivatives with regard to peptide chain length. The signal intensity values are the mean of three separate measurements.

HV capillary voltage, $-4500 \mathrm{~V}$; capillary exit, $70.0 \mathrm{~V}$; skimmer 1, 50.0 V; hexapole 1, 23.0 V; hexapole RF, $100.0 \mathrm{Vpp}$; skimmer 2, $23.0 \mathrm{~V}$; lens 1 transfer, $52.0 \mu \mathrm{s}$; and mass range, $\mathrm{m} / \mathrm{z}$ 100 - 1000. All the data acquisition and analysis were controlled using Bruker Data Analysis 3.2 software. A calibration solution of $10 \mathrm{mmol} / \mathrm{L}$ sodium formate in $50 \%$ acetonitrile was injected at the beginning of each run, and all the spectra were calibrated prior to data analysis. The data shown in the group were obtained from the mean of three separate measurements.

\section{Characterization of chemical properties of targeted peptides and} derivatives

The $\log P$ and Connolly molecular area (CMA) of the peptides were calculated using a Chem3D Pro. Ver. 19.1.0.8. software (PerkinElmer Informatics, Waltham, MA, USA).

\section{Results and Discussion}

\section{LC-ESI-MS analysis of di- to pentapeptides}

Herein, hydrophilic glycine was selected as the building block for di- to pentapeptides owing to its structural simplicity or without consideration of side chain properties. In addition to di- to pentapeptides consisting of glycine, GS, GSS, GSSS, and GSSSS with methylated peptide bonds were obtained. For chemical derivatization, four different derivatization reagents that have already been characterized to react with $N$-terminal amino groups, namely, APDS, NDA, TNBS, and 7MeO-Cou, were used. The structures of the corresponding peptide derivatives are shown in Fig. 1. The eight peptides and the corresponding 32 peptide derivatives were injected into the LCESI/MS and the MS signal intensities of all the 40 compounds were plotted against the peptide chain length (Fig. 2). Derivatization of di- and tripeptides showed a stronger response than the longer peptides overall, with $7 \mathrm{MeO}-\mathrm{Cou}$ derivatization inducing a maximum of 7-fold signal enhancement compared to non-derivatized GG. For the tetra- and pentapeptides, a maximum MS signal increase of 5-fold was observed. However, in some cases, even a decrease in the MS signal was observed for tetra- and pentapeptides. Overall, the MS signal-enhancing effects of the derivatization reagents diminished with the addition of an extra Gly or Sar. These results suggest that 
(A)

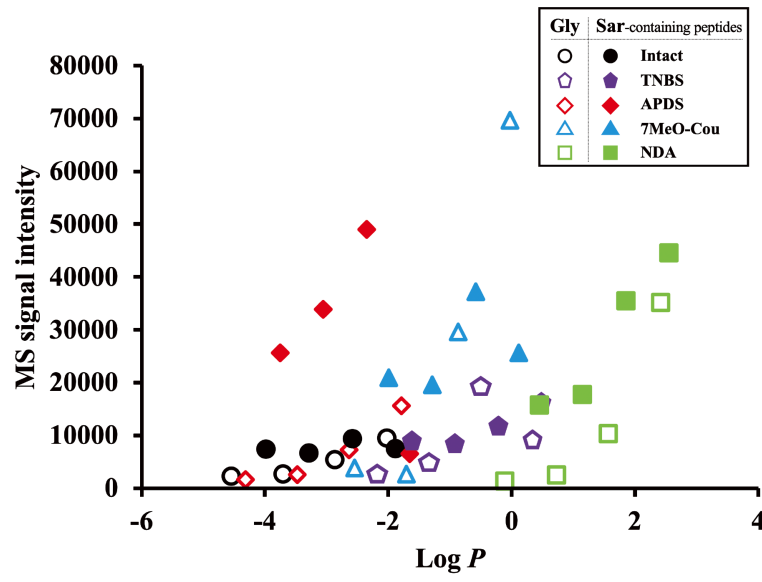

(B)

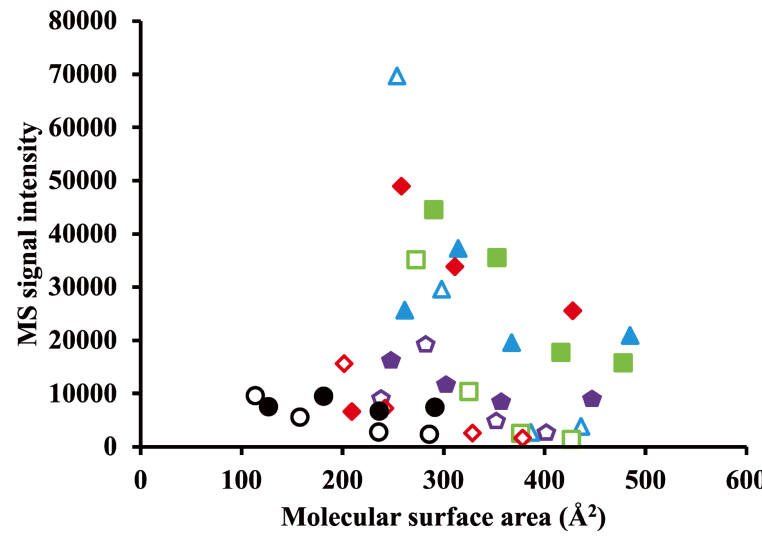

Fig. 3 MS signal intensities of 40 peptides and derivatives with regard to (A) hydrophobicity and (B) molecular surface area. The signal intensity values are the mean of three separate measurements.

chemical derivatizations might be less beneficial for longer oligopeptides, aiming to increase the sensitivity of MS detection.

\section{Effect of hydrophobicity and molecular surface area of peptides} on ESI-MS intensity

Hydrophobicity has been recognized as one of the factors affecting MS signal intensity. ${ }^{14,15}$ In this study, $\log P$ values were considered as a measure of hydrophobicity. All the four applied derivatization reagents could induce hydrophobicity increases, although to different degrees. A moderate correlation between $\log P$ and MS signal intensity was observed within individual homologous series of peptides for any of the four derivatizations (Fig. 3A). However, for individual peptides, hydrophobicity gain did not necessarily increase the MS signal intensity, and the effect was weaker for longer peptides than that for di- and tripeptides. Hydrophobic tags such as $7 \mathrm{MeO}-\mathrm{Cou}$ and NDA could be suitable for the derivatization of small diand tripeptides (Supporting Information). Meanwhile, the data in Fig. 1 show that the MS signal decreased overall as the chain length increased, suggesting that the MS signal intensity could be related to the size of the molecule itself. Therefore, we plotted the MS signal intensity against a $\mathrm{CMA}^{16}$ as a measure of the total molecular surface area. The plot of CMA and MS signal intensity showed a bell-shaped trend, where the intensity was the highest for a molecule of $200-300 \AA^{2}$. Raji et al. ${ }^{17}$ investigated the MS response of GXG tripeptides and reported a linear correlation between the MS response and molecular surface area, while the surface area of the examined tripeptides was no more than $250 \AA^{2}$. The results obtained herein suggest that the advantage of chemical derivatization might be limited as the molecule becomes larger in size. Therefore, the addition of hydrophobic tags might not be as suitable for the ESI-MS detection of longer oligopeptides as for small di-/tripeptides.

While the effect of chemical derivatization on small polar peptides has been reported, the effect on longer oligopeptides remains unclear. In this study, using peptides consisting of Gly, we demonstrated that longer peptides tend to advance less from chemical derivatization than di-/tripeptides. Herein, we evaluated the correlation of the estimated hydrophobicity $(\log P)$ or molecular surface area (CMA) of target peptides with MS signal intensity. In accordance with our results, Randall et al. ${ }^{18}$ revealed the relationship between nonpolar surface area and LC-MS response, and reported that the chemical derivatization aiming to increase the MS response was only effective for peptides with restricted sizes, and any further increase in hydrophobicity rather diminished MS signal. Therefore, it is possible that the classic strategy to increase hydrophobicity using a bulky tag might be less advantageous for longer peptides.

Herein, we synthesized oligopeptides consisting of Gly and Sar to exclude the influence of amino acid residues on MS detection. However, the effect of amino acid residues was largely neglected. GSSS and GSSSS showed stronger MS signals than GGGG and GGGGG (Figs. S1 and S2). The $\mathrm{N}$-methyl group of Sar can be attributed to an increase in the intensity. ${ }^{19}$ Interestingly, although these peptides only differ by three or four methyl groups, the response to different derivatization methods also differs between tetra-/pentapeptides consisting of only Gly and those of Gly and Sar (Figs. S1 and S2). While the methyl groups merely alter the hydrophobicity or molecular surface area of the peptides, the effect on the MS signal cannot be neglected. These results suggest that the MS signal of an oligopeptide might not be explained by chemical properties only. Moreover, the side chains could potentially post a greater effect than the addition of a moiety on one of the tails of a long peptide, rendering chemical derivatization not as beneficial as for small polar peptides. ${ }^{19}$ However, a larger panel of peptides with various side chain structures should be examined in future studies to evaluate the effect of chemical derivatization in depth.

\section{Conclusions}

Herein, we evaluated the effect of four amine derivatives on the MS signal intensities of synthetic di- to pentapeptides consisting of Gly and Sar. We demonstrated that the hydrophobicity increase induced by chemical derivatization did not result in stronger MS signals for peptides longer than di-/tripeptides. Rather, the molecular surface area of a peptide might be a determinant of the MS signal. Optimal ESI-MS detection was observed with peptides (or peptide derivatives) with a molecular surface area of $\sim 250-300 \AA^{2}$ with Gly and Sar oligopeptides.

\section{Conflict of Interest}

The authors declare no competing interests.

\section{Acknowledgements}

This study was supported in part by a JSPS Research Fellowship for Young Scientists awarded to W. S. (No. 19J20948). 


\section{Supporting Information}

The relation between MS signal intensity and $\log P$ and molecular surface area for individual peptides. This material is available free of charge on the Web at http://www.jsac.or.jp/ analsci/.

\section{References}

1. A. Iwaniak, M. Darewicz, and P. Minkiewicz, Compr. Rev. Food Sci. Food Saf., 2018, 17, 63.

2. M. Foltz, P. C. Van Der Pijl, and G. S. M. J. E. Duchateau, J. Nutr., 2010, 140, 117.

3. W. Shen and T. Matsui, Food Funct., 2017, 8, 4306.

4. T. Matsui, K. Tamaya, T. Kawasaki, and Y. Osajima, $J$. Chromatogr. B, 1999, 729, 89.

5. T. Matsui, K. Tamaya, E. Seki, K. Osajima, K. Matsumoto, and T. Kawasaki, Biol. Pharm. Bull., 2002, 25, 1228.

6. T. Matsui, M. Imamura, H. Oka, K. Osajima, K. I. Kimoto, T. Kawasaki, and K. Matsumoto, J. Pept. Sci., 2004, 10, 535.

7. T. Toyo'oka, Anal. Sci., 2017, 33, 555.

8. E. M. N. Nakashima, H.-Q. Qing, M. Tanaka, and T.
Matsui, Biosci. Biotechnol. Biochem., 2013, 77, 2094.

9. M. Tanaka, S. M. Hong, S. Akiyama, Q. Q. Hu, and T. Matsui, Mol. Nutr. Food Res., 2015, 59, 1541.

10. V. T. Hanh, W. Shen, M. Tanaka, A. Siltari, R. Korpela, and T. Matsui, J. Agric. Food Chem., 2017, 65, 5935.

11. X. Sheng, S. Nagaoka, M. Hashimoto, Y. Amiya, M. Beppu, K. Tsukamoto, E. Yanase, M. Tanaka, and T. Matsui, Food Chem., 2021, 341, 128197.

12. A. Pashkova, E. Moskovets, and B. L. Karger, Anal. Chem., 2004, 76, 4550.

13. K. Shimbo, O. Takashi, A. Yahashi, K. Hirayama, and H. Miyano, Rapid Commun. Mass Spectrom., 2009, 23, 1483.

14. N. B. Cech and C. G. Enke, Anal. Chem., 2000, 72, 2717.

15. N. B. Cech, J. R. Krone, and C. G. Enke, Anal. Chem., 2001, 73, 208.

16. M. L. Connolly, J. Appl. Crystallogr., 1983, 16, 548.

17. M. A. Raji, P. Fryčák, C. Temiyasathit, S. B. Kim, G. Mavromaras, J.-M. Ahn, and K. A. Schug, Rapid Commun. Mass Spectrom., 2009, 23, 2221.

18. S. M. Randall, I. Koryakina, G. J. Williams, and D. C. Muddiman, Rapid Commun. Mass Spectrom., 2014, 28, 2511.

19. P. Liigand, K. Kaupmees, and A. Kruve, J. Mass Spectrom., 2019, 54, 481. 